\title{
13-Year-Old Exposed Interlocking Nail: A Case Report
}

\author{
Chua YCJ, MBBS, Lim LA, MS Ortho, Hudzairy A, MS Ortho \\ Department of Orthopaedics, Batu Pahat Hospital, Batu Pahat, Malaysia
}

\begin{abstract}
We are reporting a case of post-traumatic chronic osteomyelitis of the tibia with an open wound exposing an intra medullar nail implant for 13 years. The patient presented with fresh ipsilateral tibia plateau fracture. He was treated by removal of the implant, debridement and local placement of Gentamicin-impregnated PMMA beads according to guidelines of the two-stage Belfast technique . After five months of wound treatment, the exposed bone was covered by healthy granulation tissue and the patient was able to fully bear weight.
\end{abstract}

Key Words:

Post-traumatic osteomyelitis, Chronic osteomyelitis, Interlocking nail

\section{INTRODUCTION}

Osteomyelitis is difficult to treat and cure rates for this condition remain unsatisfactory ${ }^{1}$. Post-operative osteomyelitis is a known complication and generally the implant will be removed to facilitate bone and wound healing. Harth et al. reported two cases of post-interlocking nailing osteomyelitis (infected non-union) in a series of 60 patients with tibial fractures over a 4-year period ${ }^{2}$. We are reporting a patient who presented with a closed fracture of tibial plateau and was found to have an open wound exposing an implant inserted 13 years earlier.

\section{CASE REPORT}

A 48-year-old man presented to the Emergency Department with left knee pain following a motor vehicle accident. He sustained a closed fracture of the tibial plateau. Incidentally, it was noted that there was an open wound exposing part of an intramedullary nail on the same leg. On further enquiry, the patient explained that he had undergone interlocking nail fixation for his left tibia fracture following a road traffic accident 13 years earlier. About 2 years following the injury, a wound developed over the fracture area and he noticed that the metal implant can be seen. Since the wound did not cause any significant problem to him except occasionally increase in discharge, he decided not to seek any medical attention. He was otherwise afebrile and able to fully weight-bear prior to the present admission.
Examination revealed an open wound measuring $4 \mathrm{X} 1 \mathrm{~cm}$ on the anterior aspect of the left leg with a partly exposed intramedullary nail (Figure 1). In addition, there was a discharging sinus near the ankle. The erythrocyte sedimentation rate (ESR) was $120 \mathrm{~mm} / \mathrm{hr}$ and the white cell count was $6.1 \times 1000 / \mu \mathrm{L}$. Plain radiographs of the left leg showed an intact Grosse-Kempf tibial nail with lucency around the nail suggestive of chronic osteomyelitis (Figure 2 ). Both the tibia and fibula fractures were united. A diagnosis of an acute left tibial plateau fracture and posttraumatic chronic osteomyelitis of a united tibial shaft fracture (Cierny-Mader Stage IIIA) was made.

The patient was started on empirical antibiotic therapy consisting of intravenous Cloxacillin and Fusidic Acid. During the same anaesthesia, percutaneous screw fixation of the left tibial plateau fracture was performed, followed by the removal of the existing interlocking nail, wound debridement and reaming of the medullary canal. Despite the lack of a special extraction jig, the nail was successfully removed as the screws and nail were loose. Vacuum-assisted closure (VAC) dressing was used from day 4 post-operatively and changed every three days for nine days due to copious discharge and because the patient was unable to tolerate the pain experienced during daily calcium alginate dressing. Tissue culture grew Pseudomonas aeruginosa, sensitive to Ciprofloxacin and resistant to the ß-lactam group of antibiotics. The antibiotic was then converted to parenteral Ciprofloxacin.

Subsequently, the patient underwent several surgeries for debridement, medullary reaming and wound lavage. At about one and a half months, callus was noted at the tibial plateau fracture site and the screws were removed. Gentamicinimpregnated polymethylmethacrylate (PMMA) beads were inserted during the final surgery (Figure 3A). The antibiotic beads were removed gradually over a period of twelve days before the patient was discharged with oral Ciprofloxacin. Daily wound care with calcium alginate dressing was continued.

On follow-up five months later, we noted that the wound was granulating well and covering the bone without notable discharge or inflammation of the surrounding skin (Figure 4). The patient was ambulating well without any walking aid. Eight months later, we noted minor serous discharge from 


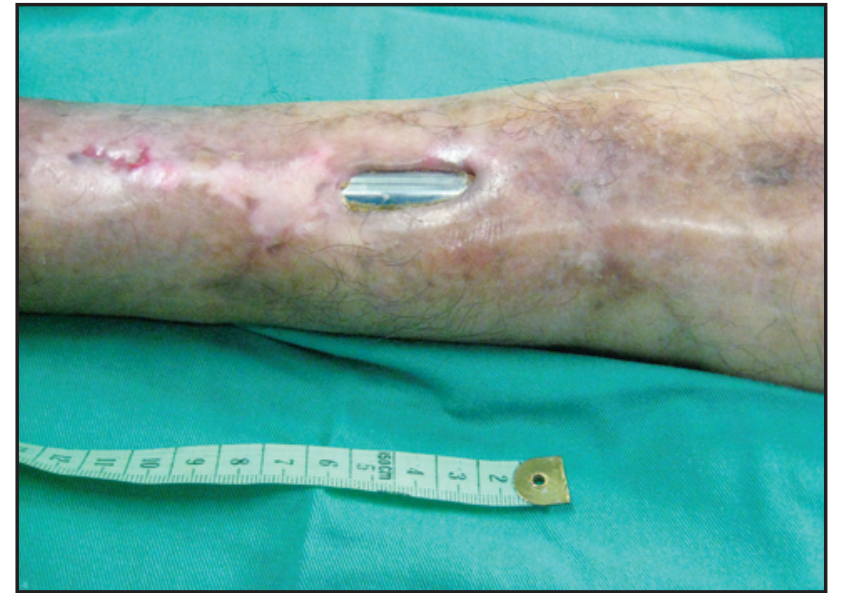

Fig. 1: Tibia interlocking nail exposed for 13 years .

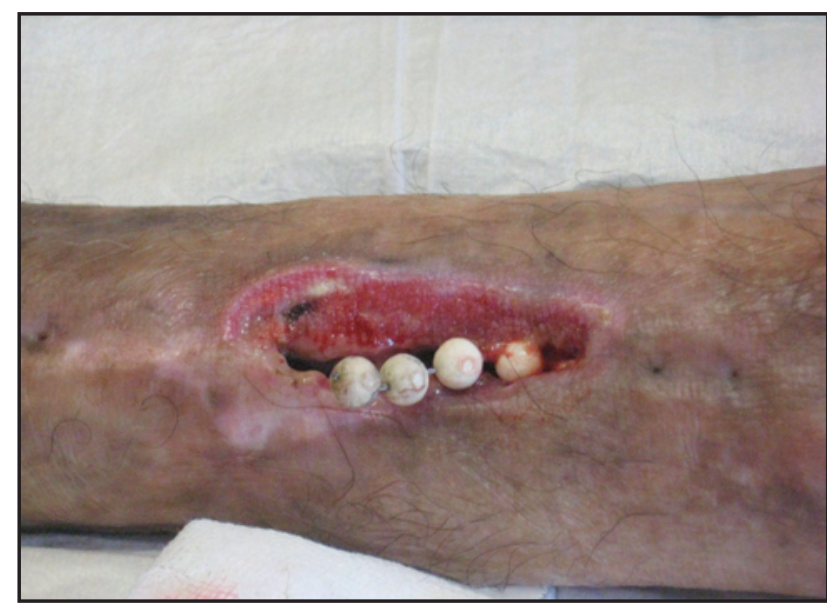

Fig. 3: Granulation tissue formed as the antibiotic beads are gradually removed from the wound.

the clean and contracting wound. The ESR remained elevated at $95 \mathrm{~mm} / \mathrm{hr}$.

\section{DISCUSSION}

There are many surgical techniques used to treat chronic osteomyelitis. The two-stage Belfast technique consists of radical debridement with an option for placement of gentamicin-impregnated PMMA beads in the defect and muscle flap if the defect is large enough and unsuitable for primary wound closure in the first stage; this is followed by removal of antibiotic beads, curettage of granulation tissue and bone grafting 3-6 weeks later, after the soft tissue has healed. A success rate of up to $91 \%$ has been reported with this technique ${ }^{3}$. The single-stage Lautenbach procedure involves debridement, medullary reaming and the insertion of double-lumen tubes into the intramedullary cavity for local antibiotic delivery and cavity analysis for volume and culture. A success rate of $94.4 \%$ has been reported with this procedure $^{4}$.

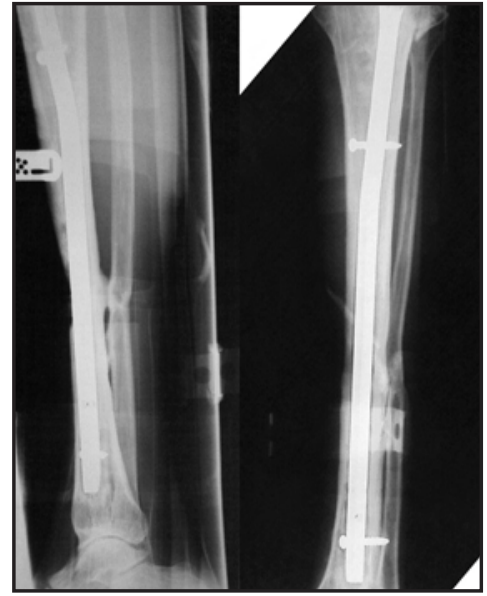

Fig. 2: Plain radiographs showing united tibia and fibula fractures with an area of bone defect just distal to the middle of the tibia.

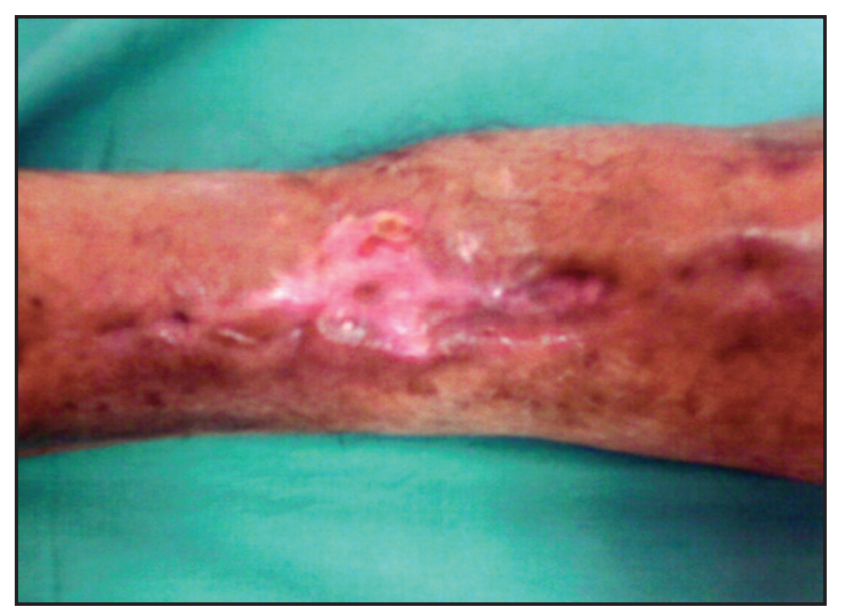

Fig. 4: The bone is covered by healthy looking granulation tissue five months later.

In the current case, we used the Belfast technique. Following the removal of the interlocking nail, multi-stage debridement, medullary reaming, and VAC dressing were performed. Subsequently antibiotic-impregnated PMMA beads were inserted and then gradually removed over a period of twelve days. Neither bone grafting nor muscle flap was performed as the patient declined a referral to a tertiary hospital. The wound was dressed on a regular basis to allow proliferating granulation tissue to cover the exposed bone. The patient was allowed to ambulate fully as the fracture was united.

Lazzarini et al. stress the high failure rate in Cierny-Mader Stage III and IV osteomyelitis without adequate debridement ${ }^{1}$. Even with adequate debridement, the remaining bed of tissue must still be considered contaminated. Medullary reaming is essential to achieve an adequate margin of debridement, especially in Cierny-Mader $\mathrm{B}$ hosts, as compared to segmental resection of an osteomyelitic lesion, which may be more suitable for CiernyMader Stage II osteomyelitis. 
utilization of a VAC dressing applies localised negative pressure over the wound surface thereby aiding in the removal of fluids ${ }^{1}$. The VAC system consists of a closed circuit of tubing connected to the wound; the tubing is covered with foam dressing and clear adhesive dressing on one end, and to a low-pressure suction device on the other end. The dressing is kept in place for at least 48 hours to allow granulation tissue to form.

Lazzarini et al. advocates complete wound closure whenever possible, using either a split-thickness skin graft for small soft-tissue defects or local muscle flaps and free vascularised muscle flaps for large soft-tissue defects in order to improve the local biological environment ${ }^{1}$. Healing by secondary intention is discouraged since the scar tissue that fills the defect may later become avascular. Another concern with such a chronic wound is malignant transformation. Although the current patient's wound was not characteristic of a Marjolin's ulcer, a tissue biopsy should be sent for histopathological examination to exclude malignancy.

When commencing oral antibiotic therapy for osteomyelitis, the drugs of proven efficacy are clindamycin, rifampin, cotrimoxazole, and fluoroquinolones such as ciprofloxacin ${ }^{1}$. Ciprofloxacin's spectrum includes both Enterobacteriaceae and $P$. aeruginosa. However, $P$. aeruginosa produces a biofilm, which may give rise to fluoroquinolone resistance. Brooun et. al. (2000) suggested three attributes of biofilm that may cause fluoroquinolone resistance, namely the presence of multidrug resistance pumps, the intrinsic resistance of bacteria growing in the biofilm, and the emergence of a "super-resistant" cell fraction as a result of the dose-dependent killing effect of antibiotic therapy ${ }^{5}$. 


\section{REFERENCES}

1 Lazzarini L, Mader JT, Calhoun JH. Osteomyelitis in long bones. J Bone Joint Surg 2004; 86-A(10): 2305-18.

2 Harth A, Moerman J, De Groote W, Vandekerckhove B, Verbeke R, Verdonk R et al. Treatment of tibial shaft fractures by interlocking nailing. Acta Orthop Belg 1993; 59(4): 381-9.

3 McNally MA, Small JO, Tofighi HG, Mollan RA. Two-stage management of chronic osteomyelitis of the long bones. The Belfast technique. J Bone Joint Surg 1993; 75-B(3): 375-80.

4 Hashmi MA, Norman P, Saleh M. The management of chronic osteomyelitis using the Lautenbach method. J Bone Joint Surg 2004; 86-B(2): 269-75.

5 Brooun A, Liu S, Lewis K. A dose-response study of antibiotic resistance in Pseudomonas aeruginosa biofilms. Antimicrob Agents Chemother 2000; 44(3): 640-6. 